\title{
Dynamic Traffic flow Control and Its Simulation on GPS Municipal Scheduling
}

\author{
Huang Guanli ${ }^{1,2}$, Zhou Meng ${ }^{1}$, Di Chunyang ${ }^{2}$ \\ ${ }^{1}$ School of Mathematics and System Science, Beihang University, Beijing 100191, China \\ ${ }^{2}$ Beijing Polytechnic, Beijing, China \\ huangguanli@smss.buaa.edu.cn
}

\begin{abstract}
This paper according the GPS technology to alleviation traffic pressure and enhance the vehicle speed, with the route choice algorithm and the GPS fluctuation vehicle intelligence transportation technology solving, realizes the way optimization. The Improved Velocity weighed Average Algorithm to further promote the realization of the decision on the status of a number of road traffic. The time frame to the distributed database for the exploration on the dynamic characteristic of transportation flowing, the newly dynamic way model to optimize the transportation constructed.
\end{abstract}

Index Terms - initial cluster centers, Traffic flow, Route optimization, Transportation Scheduling

\section{Introduction}

A good urban public transportation system is capable to ease road congestion, fuel consumption and other contaminations by attracting more people to give up private cars, which would improve the quality of living environment. The bottleneck of current road system is revealed through the analysis of data and information, which can be solved with rational use of funds. The number of motor vehicles in Beijing by 2013 is over 5.2 million, which by 2004 was only 2.5 million and was 4 million in 2010. However, some traffic facilities, such as roads, parking spots, bridges, route planning, etc., fall behind the increase of vehicles, causing traffic congestion. To solve this problem, an intelligent transportation system (ITS) was brought forward ${ }^{[1]}$.

GPS data from receivers can be depicted on the vector map by GIS technology. Technologies based on the coil and video capture are able to achieve a large number of samples and high precision, but suffer from the large-scale investment, slow implementation and limited coverage. It is unable to store and process information digitally, so as to lack means to further processing and mining. Therefore, more valuable proofs for decision making are not available ${ }^{[2]}$. Real-time road conditions are achieved with radio detection and location system, by collecting cellphone signals via wireless collection instruments and calculating the velocity of the cellphone user. Due to the low coverage of base stations and their poor location accuracy, and the fluctuation of wireless signals, a large amount of data is required for calculation, which limits its application significantly. Therefore, the results from these two methods are not precise or timely enough, which fail to guide vehicles in real-time to ease urban traffic pressure.
GPS floating car technology is capable to get real-time traffic information with a large coverage and high precision, based on the GPS acquisition module installed in vehicles. The combination of GPS navigation system with electronic map, radio communication network and computer management information system, is able to achieve vehicle tracking, traffic management and many other applications.

\section{Combination of GPS Technology with Path Optimization Algorithm based on Floating Car Data}

A. Design of dynamic path optimization control algorithm in the urban traffic scheduling

A vector describing velocity, position, direction and other states, is used to represent a single vehicle, so that states of all vehicles in an observed section are described by a variable sequence. Assuming that it is capable for all vehicles to transfer their states through GPS technology, the dynamic sequence generates a dynamic target stream within a certain observation space. The characteristic of the target stream is analyzed and its dynamic path optimization model is established, to simulate the change of traffic and determine or predict the probability of congestion in an observation point at a future time.

The reliability of the following investigations and simulation results is dependent on the rationality and validity of the model. As the city with the fastest development of ITS, a large number of traffic data have been accumulated in Beijing, and various collecting methods developed as well. Moreover, the development of the Internet and mobile communication technology and the increase of the installed capacity of in-vehicle GPS also provide material conditions for data real-time collection and centralized processing. The validation of the model can be achieved by analyzing these data. Initial parameters of the model are estimated from real-time collected data. Then, the solution of the model is calculated in real time by computer simulations, so as to determine or predict the probability of congestion in an observed point at a future time. As the non-congested sate is assumed to a zero drift state, there is a dynamic drift of the congested state inevitably. Assuming that the dynamic node and condition are controllable, the control scheme of the congestion is solved by controlling the drift through optimization control algorithm. The influences of the changes of state, such as traffic lights control, traffic diversion, speed limit, road planning, and road reconstruction, on the alleviation of the traffic 
congestion are obtained respectively, with variations of the control condition.

\section{B. Analysis of traffic flow characteristic and setup of a novel model}

In the Free Flow phase $(\mathrm{F})$, the speeds of vehicles maintain maximum, and vehicles have no direct connection with each other, only affected by the vehicles themselves and the drivers. However, the phase will change as the capacity of a specific section is lower than other sections significantly due to some dynamic factors (such as fault, accident, etc. $)^{[3]}$. In the Congested Flow phase, the speeds of vehicles are lower than the minimum of the feasible average speed in the Free Flow phase significantly, and there is a strongly nonlinear interaction between vehicles, which shows a complicated dynamic space-time characteristic. This phase can be further divided into two phases, Synchronized Flow phase (S) and Jams Flow phase (J). The flow rate is still high in the Synchronized Flow phase, but the average speed is low and the density is high. This phase has the trend to achieve the synchronized speed for vehicles in one lane or multi-lane by crossing lanes. There is no obvious stopping area of vehicles in the space or time within the phase. In other words, all vehicles are moving forward under the strong interaction. Most downstream fronts of the Synchronized Flow are fixed at the bottleneck. Besides, the downstream fronts of the moving Synchronized Flow are also fixed at the bottleneck, as the mode is always captured by the traffic bottleneck when passing through it, which is called the Catch Effect.

Synchronized Flow is divided into three states further. The first is the steady and uniform Synchronized Flow state, the speed, density and flow rate of which are approximately uniform and constant in a long time (2-5 minutes). The second state is defined for the condition when the average speed is still approximately uniform and constant in a long time, but the density and flow rate change significantly. In this state, the speeds of vehicles in different lanes can not achieve the synchronized trend. Finally, in the third state, the speed, density and flow rate are all neither uniform nor constant, and obvious changes exist between adjacent experimental data points. All three sub states of Synchronized Flow are two-dimension distributed in the flow rate-density plane, the first two of which are rare and the third is common[4]. The Synchronized Flow phase is likely to transfer to the Jams Flow phase with dynamic disturbance.

The Jams Flow phase is a local structure moving towards the upstream, restricted by two fronts with vehicle speed mutation. The distance between the two fronts is much larger than the range of the front. And the speed and flow rate in this phase is nearly zero. It is capable to pass through the Free Flow, Synchronized Flow and any bottlenecks without being captured, and the downstream front keeps transmitting towards upstream in an approximately constant speed. It is benefit for transferring to the Synchronized Flow phase, by controlling the movement of nodes near the fronts rationally, to ease congestion [5].

\section{Definition and Calculation Design of Dynamic Optimization Path}

To solve the problem of the traffic congestion further, based on the analysis of the floating car collected data and the integration of the Petri net and other traffic flow theoretical models[6], a three-phase dynamic target flow model is established to describe the traffic flow state and the phase change mechanism for the optimized control of traffic flow. A path optimization algorithm combined GPS and GIS technology is designed grounded on the floating car collected data, which describes the traffic condition digitally and quantitively and displays it on the electronic map. Then, the topological structure between the origin and the destination is abstracted, according to traffic route data on the electronic map. Finally, the path optimization algorithm based on the graph theory is adopted to calculate the shortest path on the above topological structure, with the practical calculation example presented.

Take the real traffic data (roads, control, vehicles, etc.) of a specific road section, section of Beijing HuiLongguan residential, as an example. The change of the probability of microscopic interaction among traffic flows is set as the trigger condition of the system state. The mathematical model can be determined, with initial parameters of the variable sequence, state space and probability transform matrix[7] in a specific section estimated. Sequentially, the probability of the congestion in an observed point at a time can be determined or predicted by solving the mathematical model. With the investigation on the optimized control algorithm of the dynamic target flow, the optimized control solution is figured out on the real data. Therefore, the change of the dynamic target flow is under control to achieve the inverse transform from the Jams phase to the Free Flow phase, for the ease of traffic congestion.

The speeds of cars in the traffic lines of the city can be obtained with a sufficient number of floating cars, denoted as v. Mark the speed on the GIS map with three different lines, corresponding to the state of congested, postponed and smooth. The sketch map of many bottleneck road sections within different districts in Beijing is shown in Figure 1. Meanwhile, the distance of any two points in the map can be obtained as well, denoted as $s$. The time $t$, in which a car is driven through distance $\mathrm{s}$ with speed $\mathrm{v}$, is marked on the topological map of the road, as the path coefficient of the route.

\section{Implementation of the Dynamic Optimal Path Algorithm}

\section{A. Description of the problem of the optimal path algorithm}

The shortest path problem is a classical algorithm problem in the graph theory, aimed at finding the shortest path between two nodes in a figure composed of nodes and paths.

Nodes are represented by numbers with circles, 
corresponding to intersections of the road network, such as crossroads, closed road crossing gates, etc., and distances or times between two points are represented by directional lines between two nodes. With two points in the figure given as the origin and destination respectively and the shortest distance between the two set as the control rule, the shortest path is able to obtain via a certain algorithm performed on the entire map.

\section{B. Selection and description of the algorithm}

To solve the shortest path, the conventional algorithms mainly are Dijkstra algorithm, A* algorithm, SPFA algorithm, Bellman-Ford algorithm, Floyd-Warshall algorithm, Johnson algorithm and genetic algorithm. Dijkstra algorithm is investigated in this subject.

Dijkstra algorithm is a typical one for calculating the shortest path from a node to any other nodes. The main characteristic is to set the original point as the center and expand outwards until to the destination. Though Dijkstra algorithm is capable to achieve the optimal solution of the shortest path, the efficiency is quite low due to the large number of nodes it traverses, the calculation complexity of which is $O\left(n^{\wedge} 2\right)$.

\section{Modeling of Dijkstra Algorithm}

Assuming $\mathrm{n}$ vertices in the map, the set $\mathrm{Ei}$ is an one-dimension array, composed of $n$ weights from the ith vertex to all vertices. The weight from the vertex $i$ to itself is 0 , and the weight from it to a vertex without direct link between the two is $\infty$. A larger value is taken in practice, such as $E 1=\{0,1,6,3,4,3,1, \infty, \infty, \infty, \infty, \infty, \infty\}$ in Figure 3. All weight sets of $n$ vertices compose a matrix En $\times n$, denoted as $E$ to represent the entire map. For the sake of calculation facility, the original point is defined as the 0th point, and the destination defined as the $\mathrm{n}-1$ th point.

The set $\mathrm{S}$ described states of $\mathrm{n}$ vertices needs recorded in the calculation, the elements of which represent the vertices involving into the current path calculation. As there is only the original point in the calculation initially, we set
$\mathrm{S}[0]=1$ and $\mathrm{S}[\mathrm{i}]=0$ for vertices not in the calculation. It is the shortest distance set from the original point to all vertices, Distance, corresponding to $\mathrm{S}$. At the initial state, distance $[i]=E[0, i](i=0, \ldots, n-1)$, referring to the matrix $E$, and apparently Distance[0]=0. The Distance value of the vertex adjacent to the original point is the weight from it to the vertex, while the value of the vertex not adjacent to the original point is infinite.

To record the shortest path from the original point to any other vertices, the set Path is defined, with Path[i] representing the shortest path from the original point to the ith vertex. For example, $\operatorname{Path}[\mathrm{i}]=(0,1,3$, i), demonstrates that the shortest path from the original point to the ith vertex is achieved via vertices 1 and 3 . At the initial state, Path is determined by the adjacent matrix of the original point. If $\mathrm{E}[0, \mathrm{i}]$ is finite, the index of vertex $\mathrm{i}$ is stored into the Path[i] together with the index of the original point, which is Path $[\mathrm{i}]=(0, \mathrm{i})$.

The calculation is accomplished with n-2 times. Sets S, Distance and Path are initialized at first. Then, the vertex corresponding to the shortest distance is set as the middle point for calculation, after comparing all distances with $\mathrm{S}[\mathrm{j}]=0$ in the set Distance. For example, if Distance[i] is the shortest, the vertex $i$ is added into the set for calculating the distance. By setting $\mathrm{S}[\mathrm{i}]=1$, amend the Distance[j] and Path $[\mathrm{j}]$ for all vertices $S[\mathrm{j}]=0$. The procedure is performed as follow: comparing the current Distance[j] and Distance $[\mathrm{i}]+\mathrm{E}[\mathrm{i}, \mathrm{j}]$, if Distance[j] $<=$ Distance $[\mathrm{i}]+\mathrm{E}[\mathrm{i}, \mathrm{j}]$, no update is performed, otherwise, set Distance $[\mathrm{j}]=$ Distance[i]+E[i,j] and $\operatorname{Path}[\mathrm{j}]=(0, \mathrm{i}, \mathrm{j})$. The above step is repeated until all vertices in $\mathrm{S}$ are indexed as 1 . At this time, the shortest distances from the starting point to any other point are stored in Distance, while the shortest paths are stored in Path.

Set the vertex as the middle point for calculation, as its distance is the shortest. S, Distance and Path are calculated as follow table I:

TABLE I Distance and Path of Shortest

\begin{tabular}{|c|c|c|c|c|c|c|c|c|c|c|c|c|c|}
\hline Vertex & 0 & 1 & 2 & 3 & 4 & 5 & 6 & 7 & 8 & 9 & 10 & 11 & 12 \\
\hline $\mathrm{S}$ & 1 & 1 & 0 & 0 & 0 & 0 & 1 & 0 & 0 & 0 & 0 & 0 & 0 \\
\hline $\mathrm{D}$ & 0 & 1 & 6 & 3 & 4 & 3 & 1 & 7 & 7 & $\infty$ & $\infty$ & $\infty$ & $\infty$ \\
\hline $\mathrm{P}$ & & 0,1 & 0,2 & 0,3 & 0,4 & 0,5 & 0,6 & $0,1,7$ & $0,6,8$ & & & & \\
\hline
\end{tabular}

The final results are given here as table II:

TABLE II Final Results of Distance and Path

\begin{tabular}{|c|c|c|c|c|c|c|c|c|c|c|c|c|c|}
\hline & 0 & 1 & 2 & 3 & 4 & 5 & 6 & 7 & 8 & 9 & 10 & 11 & 12 \\
\hline $\mathrm{S}$ & 1 & 1 & 1 & 1 & 1 & 1 & 1 & 1 & 1 & 1 & 1 & 1 & 0 \\
\hline $\mathrm{D}$ & 0 & 1 & 6 & 3 & 4 & 3 & 1 & 7 & 7 & 11 & 16 & 9 & 21 \\
\hline $\mathrm{P}$ & & 0,1 & 0,2 & 0,3 & 0,4 & 0,5 & 0,6 & $0,1,7$ & $0,6,8$ & $0,3,9$ & $0,6,8,10$ & $0,3,11$ & $0,3,11,12$ \\
\hline
\end{tabular}

It is shown from the table, the shortest path from the distance 21 . vertex 1 to 13 is achieved via 4 and 12, with the shortest 


\section{Summaries and Outlooks}

In this paper, the concept of three-phase dynamic traffic flow is proposed initially, which breaks the limit of the traditional traffic flow theory, and provides an effective complement to the theory. Integrating various models of traffic flow, a reformed traffic flow model is established, which is a major innovation in this field. Many theories and technologies, such as artificial intelligence, operation research, computer simulation, GPS and wireless communication, are applied in the research of traffic flow, which breaks the limit of the traditional study. With the traffic flow model fit for the domestic situations established, the transmission from the theory to the practice is successfully achieved, which provides a novel access to solving the urban traffic problem. The application of intelligent optimized control, computer simulation and real-time GPS information, opens up a new idea and method for traffic flow modeling and controlling, which is capable to solve the problem of urban traffic congestion. The combination of the traffic flow and real in-vehicle GPS data also sets up a novel urban traffic flow model and breaks a new path. Based on the real-time state of traffic flow, the optimal control path is calculated in real time and released to the relative nodes. Therefore, the load of network traffic flow is balanced and the congestion is eased timely, which improves the efficiency significantly. Based on the technologies of computer simulation, operation research, and artificial intelligence, an approach to detect, analyze and determine the congested nodes in real time and an optimized control algorithm of dynamic traffic flow are proposed, respectively. They contribute to the development of urban ITS, by achieving the real-time determination and optimized control of traffic state, balancing the load of the urban traffic flow and easing the traffic congestion.

\section{References}

[1] Blanco A, Delgado M, Pegalajar M C. A real-coded genetic algorithm for training recurrent neural networks. Neural Networks, 2001, 14, 93-105

[2] Baldea Michael, Daoutidis Prodromos. Model reduction and control of reactor heat exchanger networks. Journal of Process Control, 2006, 16(3), 265-274

[3] Christos G Cassandras, Gang Sun, et al. Perturbation Analysis and Control of Two-Class Stochastic Fluid Models for Communication Networks. IEEE Transactions on Automatic Control, 2003, 48(5), 770-782

[4] Gifidhar A, Kumar P R. Scheduling Automated Traffic on a Networks of Roads. IEEE Transactions on Vehicle Technology, 2006, 55(5), 1467-1474

[5] [5]Nelly Barbot, Bruno Sericola. Distrbution of Busy Period in Stochastic Fluid Models. Communications in Staristics-Stochasitc Models, 2001, 17(4), 407-427

[6] Smith B L,et al. Comparison of parametric and nonparametric models. Transportation Research Part C, 2002, 10, 303-321

[7] Verikas A, Bacauskiene M. Using artificial neural networks for process and system modeling. Cheemometrics and Intelligent Laboratory Systems, 2003, 67, 187-191 